\title{
Early Detection of Central Pontine Myelinolysis with MRI: A Case Study
}

\author{
Goutam Chowdhury \\ Abdul Ahad Mohammed Ryhan Uddin ${ }^{2 *}$ \\ Md Abdur Rouf ${ }^{3}$ \\ Rajat Sanker Roy Biswas ${ }^{4}$ \\ Md Ismail ${ }^{5}$ \\ Md Siraz ${ }^{6}$ \\ Mohammed Musfequr Rahman \\ 'Department of Radiology and Imaging \\ Chevron Clinical Laboratory \\ Chittagong, Bangladesh \\ ${ }^{2}$ Department of Medicine and Critical Care Unit \\ University of Science \& Technology Chittagong \\ Chittagong, Bangladesh.
}

${ }^{3}$ Department of Medicine and Cardiology University of Science \& Technology Chittagong Chittagong, Bangladesh.

${ }^{4}$ Department of Medicine

Chattagram International Dental College \& Hospital International Islamic Medical College \& Hospital Chittagong, Bangladesh.

${ }^{5}$ Department of Dermatology University of Science \& Technology Chittagong Chittagong, Bangladesh.

${ }^{6}$ Department of Medicine University of Science \& Technology Chittagong Chittagong, Bangladesh.

${ }^{7}$ Department of Anaesthesia Chittagong Medical College Chittagong, Bangladesh.

*Correspondence to:

Dr. Abdul Ahad Mohammed Ryhan Uddin Department of Medicine and Critical Care Unit University of Science \& Technology Chittagong Chittagong, Bangladesh.

Mobile: +8801817744297

E-mail: ryhandr@yahoo.com

\begin{abstract}
Central pontine myelinolysis (CPM) is a complication of treatment of patients with severe hyponatremia. The microscopic appearance of central pontine myelinolysis is loss of myelin with sparing of axons, without evidence of inflammation. The abnormalities associated with central pontine myelinolysis are readily identified on magnetic resonance imaging scans. A patient with central pontine myelinolysis following rapid correction of hyponatremia was studied with magnetic resonance imaging soon after onset of tetraplegia \& dysarthria. Affected central pontine white matter showed restricted diffusion on diffusion-weighted images associated with hyper signal on T2-W \& FLAIR images. MRI with dedicated sequences is the modality of choice for early detection of osmotic changes in the brain.
\end{abstract}

Key words: Magnetic resonance imaging; central pontine myelinolysis; hyponatremia.

\section{INTRODUCTION}

Central pontine myelinolysis $(\mathrm{CPM})$ is an acquired toxic demyelinating disease (osmolar disturbance) and occurs as a consequence of certain metabolic derangements. These include rapid correction of hyponatremia, as well as hyperosmolar conditions, such as hyperglycemia. It reportedly occurs in chronic alcoholics, malnourished, transplant recipients, chronically debilitated patients, pregnancy, and burn. CPM is caused by the destruction of the medullated sheath in the brainstem (pons), preserving the axons and neurons ${ }^{1}$. Patients typically experience a rapidly evolving paraparesis, quadriparesis, pseudobulbar symptoms such as dysarthria and dysphagia. They may present with disturbed level of consciousness, gait disturbance and 'locked-in syndrome'2. Magnetic resonance imaging (MRI) with diffusion weighted imaging (DWI) is the modality of choice for early diagnosis of this entity. This study was done using 1.5 Tesla superconductive MR unit at department of Radiology \& Imaging, Chevron Clinical Laboratory (Pte) Ltd, Chittagong.

\section{Case presentation}

A 35 year-old female patient admitted because of decreased sensorium. Three days before admission she had visited a local clinic due to persistent vomiting and diarrhea for two days which culminated in hyponatremia (serum sodium $102 \mathrm{mmol} / \mathrm{L}$ ) and hypokalemia (serum potassium $3.2 \mathrm{mmol} / \mathrm{L}$ ). Her condition improved for a day, after a two-day vigorous sodium replacement with hypertonic and normal saline, together with fluid restriction converted the serum sodium to $126 \mathrm{mmol} / \mathrm{L}$ (increase of 12 mmol per day), and shortly followed by reversal to a semiconscious state. Rapid I/V infusion continued in the local clinic. However on the day before admission, her level of consciousness decreased to a drowsy state and she became dysarthric \& tetraplegic on the day of admission. According to the clinical data of intensive care unit (ICU) where she was admitted, she was comatose without eye opening, verbal output, or motor response. Abdominal radiograph showed paralytic ileus, secondary to electrolyte imbalance. According to the neurologist blood tests including serum electrolytes and a cranial computed tomography (CT) scan was advised. 
Initial CT scan did not show any low attenuated areas. Immediate MRI of the brain was advised and requested to be done on a superconductive MRI unit under full supervision of MR radiologist with all necessary sequences. MR imaging was done applying dedicated pulse sequences including DWI. The DWI (TR $=3500 \mathrm{~ms} \&$ TE $102 \mathrm{~ms})$ shows a trident-shaped high signal area at central pons (Fig. 3) that is dark on apparent diffusion coefficient (ADC) (Fig. 4). The diffusion restricted area was hyper signal on T2-Weighted spin-echo $(\mathrm{TR}=4010 \mathrm{~ms} \& \mathrm{TE}=107 \mathrm{~ms})($ Fig. 1$)$ and fat-sat fluid attenuation inversion recovery $(\mathrm{TR}=9000 \mathrm{~ms} ; \mathrm{TE}=96 \mathrm{~ms} \&$ $\mathrm{TI}=2500 \mathrm{~ms}$ ) images (Fig. 2), no appreciable altered signal on T1-Weighted spin-echo $(\mathrm{TR}=500 \mathrm{~ms} \& \mathrm{TE}=9.4 \mathrm{~ms})$ image . No evidence of hemorrhage seen on gradient echo (GRE) images $(\mathrm{TR}=800 \mathrm{~ms} ; \mathrm{TE}=26 \mathrm{~ms})$. There was no extrapontine abnormal signal. No intravenous contrast medium (Gd-DTPA) was given. The sodium level was at $155 \mathrm{mmol} / \mathrm{L}$ at that period. The patient had improvement in clinical condition upon hospital discharge.
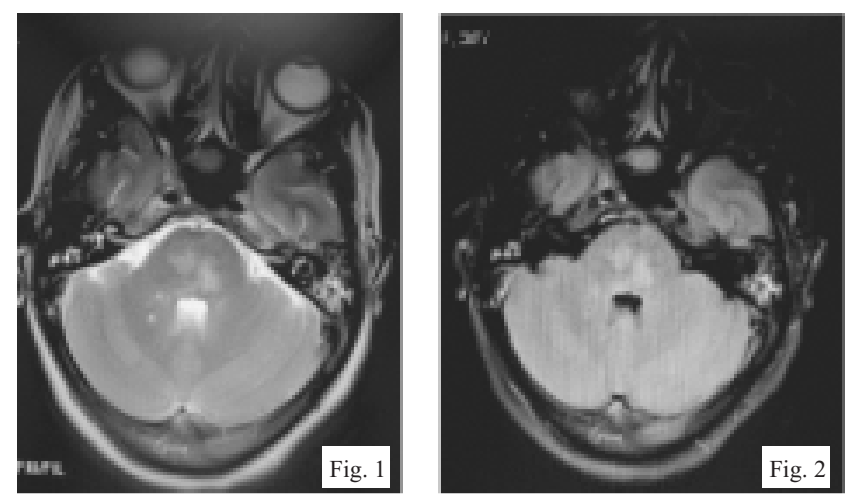

Figures 1 \& 2 : Axial-T2W and FLAIR MR scans showing hyperintense signal in the central pons.
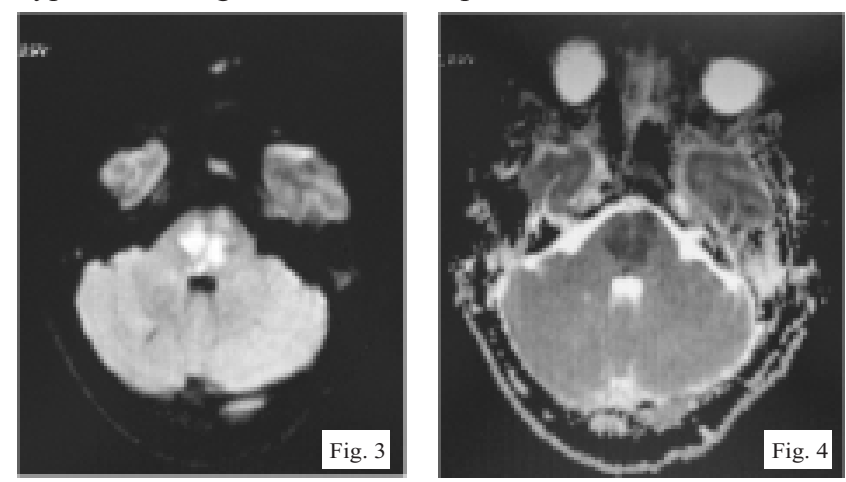

Figures 3 \& 4: Corresponding Axial DWI showing restricted diffusion in the central pons.

\section{DISCUSSION}

Central pontine myelinolysis (CPM) was first termed in 1959 by Adams and colleagues to describe a symmetric, demyelinating focus prominent in the central pons affecting the alcoholics and malnourished ${ }^{3}$. The concept was extended from 1962 with the recognition that lesions can occur outside the pons, so-called extrapontine myelinolysis (EPM). In 1976 a link between these disorders and the rapid correction of sodium in hyponatremic patients was suggested, and by 1982 substantially established ${ }^{4}$.
Three sub-types of osmotic myelinolysis have been described: (1) Central pontine myelinolysis (CPM), in which the lesion is confined to the pons; (2) extra pontine myelinolysis (EPM), in which the lesions are confined to the basal ganglia, mid brain, thalami, cerebral subcortical white matter, and cerebellum; and (3) osmotic demyelination syndrome (ODS), in which the $\mathrm{CPM}$ and EPM lesion sites are both present . ${ }^{2} \mathrm{CPM}$ is more common than combined CPM and EPM, or isolated EPM. In a study by Gocht and Colmant on 58 cases, 27 had CPM alone, 18 had combined CPM and EPM, whilst 13 presented with $\mathrm{EPM}^{5}$.

CPM has traditionally been associated with rapid correction of hyponatremia. Alcoholism, chronic malnutrition, and sodium imbalances are the primary conditions reported with CPM. More recent studies have implicated rapid correction of hyponatremia and hypernatremia as the cause of CPM or ODS. Hypokalemia in association with hyponatremia has also been implicated ${ }^{2}$. Over $75 \%$ of cases are associated with chronic alcoholism or rapid correction of hyponatremia ${ }^{6}$.

It is a condition characterized by symmetrical loss of myelin in the central pons, spreading centrifugally. In severe cases, necrosis and cavitation may be seen, and extension to tegmentum and midbrain can occur. When it occurs sometime in extrapontine regions, it is termed EPM, notably in the basal ganglia, thalami, cerebral and cerebellar gray-white junctions. CPM and EPM may occur alone or in conjunction?

Histologic studies have shown oligodendroglial cells are most susceptible to CPM-related osmotic stresses, with the distribution of CPM changes paralleling the distribution of oligodendroglial cells that normally embed large neurons within the central pons, thalamus, cortex, putamen, lateral geniculate, and other extrapontine sites. The transverse pontocerebellar fibers are most frequently involved, followed by long rostrocaudal tracts. Histologically, there is preservation of neurons and axons, differentiating this process from a central pontine infarct. There is usually no inflammatory reaction associated with osmotic demyelination, differentiating the process from multiple sclerosis, which is characterized by marked perivascular inflammation ${ }^{8}$.

Diagnosis of CPM is based on clinical suspicion and is confirmed by imaging studies ${ }^{2}$. CPM can be seen on CT as symmetric hypodense areas, but MRI is frequently striking and is the imaging technique of choice, having a greater sensitivity for CPM than CT and superior capacity for the demonstration of the lesions of EPM.

MR imaging findings of CPM include symmetric hyper signal intensity focus in the central part of the pons with an unaffected outer rim at T2-weighted and fluid attenuation inversion recovery (FLAIR) imaging ${ }^{9}$. This may progress to classic hyperintense "trident or Mexican hat-shaped" central pontine abnormality, with sparing of the ventrolateral pons and corticospinal tracts. There is associated decreased $\mathrm{T} 1$ signal intensity without mass effect. The distribution of the imaging findings mirrors the demyelination pathophysiology ${ }^{8}$. Enhancement has been reported from none to be faintly homogeneous and peripheral ${ }^{7,9}$. It is not uncommon for MRI to be negative in patients with suspected ODS up to 10 days after the onset of clinical symptoms. 
Magnetic resonance diffusion weighted imaging (DWI) is a relatively new MR technique sensitive to the motion of water. Because the underlying process of CPM is the osmotic disturbance of water and electrolyte imbalance, it follows that DWI may be a better method to identify early pathophysiologic change. Current literature demonstrates DWI to be sensitive at very early time points after ischemic injury. Restricted diffusion may be the first imaging manifestation of CPM, occurring within 24 hours of clinical onset of tetraplegia and before detection of abnormalities on conventional MR images, including SE T1-weighted, SE T2-weighted, and FLAIR images $^{8}$. Lesions on MRI may appear days to weeks after the onset of symptoms and may resolve, in some cases, over a period of months ${ }^{2,7}$.

Differential diagnosis of central pontine myelinolysis includes perforating basilar artery infarct, metastasis, glioma, multiple sclerosis, encephalitis, radiotherapy and chemotherapy ${ }^{10}$.
To prevent osmotic myelinolysis, gradual, controlled treatment of low sodium levels may reduce the risk of nerve damage in the pons. There is recommendation of correcting hypokalamia in patients prior to correcting hyponatrimia. Overcorrection of hyponatrimia (greater than $18 \mathrm{mmol}$ of sodium per 24 hours, sometimes as low as $10 \mathrm{mmol}$ per 24 hours or $21 \mathrm{mmol}$ per 48 hours) is to be avoided. ${ }^{7}$ Hyponatremia should be corrected at a rate of no more than $8-10 \mathrm{mmol} / \mathrm{L}$ of sodium per day to prevent central pontine myelinolysis.

\section{CONCLUSION}

Central pontine myelinolysis or osmotic demyelination syndrome is most often found in patients with electrolyte abnormalities, especially rapidly corrected hyponatremia. Early diagnosis with MRI using diffusion weighted imaging may demonstrate changes of CPM/ODS allowing for earlier medical intervention and a favorable outcome.

\section{DISCLOSURE}

All the authors declared no competing interest.

\section{REFERENCES}

1. Jae KR, Hyunwoo N, Myung CL. A case of central pontine and extrapontine myelinolysis with early hypermetabolism on ${ }^{18}$ FDG-PET scan. J Korean Med Sci 1998; 13: 99-102.

2. Musana AK, Yale SH. Central pontine myelinolysis: case series and review. Wisconsin Medical Journal 2005;104 (6): 56-60.

3. Adams Raymond D, Victor Maurice, Mancall Elliott L. Central pontine myelinolysis: a hitherto undescribed disease occurring in alcoholics and malnourished patients. A. M. A. Arch Neurol Psychiatry 1959;81(2):154-72.

4. Martin RJ. Central pontine and extrapontine myelinolysis: the osmotic demyelination syndromes. J Neurol Neurosurg Psychiatry 2004;75:iii22-iii28

5. Gocht A, Colmant HJ. Central pontine and extrapontine myelinolysis: a report of 58 cases. Clin Neuropathol 1987; 6(6): 262-70.

6. Osborn AG. Diagnostic Neuroradiology. Mosby, 1994;761-763.

7. Huin E, Tan KP. CT and MR findings in central pontine and extrapontine myelinolysis: A study of two patients. Singapore Medical Journal 1996; 37:622-626.

8. Kimberly AR, Norbert GC, Gary MM. Early diagnosis of central pontine myelinolysis with Diffusion-Weighted Imaging. AJNR Am J Neuroradiol 2004; 25:210-213.

9. Monika BF, Tomasz B, Irena R et al. MR imaging of seven presumed cases of central pontine and extrapontine myelinolysis. Acta Neurobiol Exp 2001; 61: 141-144.

10. Miller GM, Baker HL jun.., Okozaki H, Whisnant JP. Central pontine myelinolysis and its imitators: MR findings. Radiology $1988 ; 168: 795-802$. 